\title{
Combinatorial and Automated Proofs of Certain Identities
}

\author{
Justin Brereton \\ Massachusetts Institute of Technology \\ jbrere@MIT.EDU \\ Maryam Karnib \\ Wayne State University \\ maryamk24@gmail.com \\ Alex Quenon \\ Eastern Michigan University \\ altheuser@gmail .com
}

\author{
Amelia Farid \\ Columbia University \\ amf2153@columbia.edu \\ Gary Marple \\ Colorado State University-Pueblo \\ marbleandmarple@hotmail.com \\ Akalu Tefera \\ Grand Valley State University \\ teferaa@gvsu.edu
}

Submitted: Apr 29, 2011; Accepted: Jun 8, 2011; Published: Jun 18, 2011

Mathematics Subject Classification: 05A19

\begin{abstract}
This paper focuses on two binomial identities. The proofs illustrate the power and elegance in enumerative/algebraic combinatorial arguments, modern machineassisted techniques of Wilf-Zeilberger and the classical tools of generatingfunctionology.
\end{abstract}

Key Words and Phrases: recurrence equations, combinatorial identities, Zeilberger Algorithm, WZ, generatingfunctionology.

Dedicated to Doron Zeilberger on the occasion of his sixtieth birthday.

\section{Introduction}

Conjecturing and proving identities of the form $A=B$ (where $A$ is sum of 'nice' terms (such as binomial) and $B$ is a closed form or a sum of 'nice' terms) is among ancient and attractive mathematical problems. There are several types of proof techniques from 
various areas of mathematics that can be used to prove such identities. Among these techniques, we mention three here: enumerative combinatorics, generatingfunctionology and the the Wilf-Zeilberger (WZ) method. Enumerative combinatorics deals with counting the number of certain combinatorial objects. It gives meaning and understanding of such objects, and provides an elegant and creative way of verification. Many problems that arise in applications have a relatively simple combinatorial interpretation. For example the binomial coefficient $\left(\begin{array}{l}n \\ k\end{array}\right)$ counts the number of different ways of selecting $k$ objects from a set of $n$ objects. Even though this method gives combinatorial interpretations, it is at times challenging to find combinatorial descriptions for identities that have multiple parameters or involve non-integral values. For an introduction on combinatorial argument techniques, see, among others, [1,2]. Another classical method for proving identities is the generatingfunctionology technique which is time-tested since Euler. It uses formal series expansions and is flexible enough to be applied to many situations. Formal series expansions satisfy additive and multiplicative properties making them one of the most widely used methods for proving identities. However, this approach involves tedious algebraic manipulations and lacks to give meaning of the identities. A classical book that discusses the generatingfunctionology method is [7]. The WZ method is one of the most recent, efficient, computer-assisted/automated revolutionary technique for proving identities. Among its powerful features are its instant generation of elegant and short proofs and its ability to validate identities for non- integral values of free parameters (such as, real, complex, even indeterminates) as well as broader generalizations. For a superb exposition of the WZ method see, among others, the books [5, 4] which are devoted to this and other methods. For an abridged (10 minutes) introduction to the WZ proof style see [6].

In this paper we present proofs of two identities using the above mentioned methods. The paper is organized as follows. In Section 2 we state the main identities. In Section 3 we provide combinatorial proofs. In Section 4 we present computer generated proofs of the main identities. In Section 5 we provide proofs of the identities using generating functions. Throughout this paper we denote the set $\{k, k+1, k+2, \ldots\}$ for $k \in \mathbb{Z}$ by $\mathbb{N}_{k}$. We use the convention that $\left(\begin{array}{l}a \\ b\end{array}\right)=0$ for $b<0$ or $a<b$. For a formal power series $F(x)=\sum_{n=0}^{\infty} f_{n} x^{n}$, we denote the coefficient of $x^{n}$ by $\left[x^{n}\right] F(x)$.

\section{Main Identities}

Theorem 1. If $n, m \in \mathbb{N}_{0}$ then

$$
\sum_{r=0}^{m} 2^{n-r}\left(\begin{array}{l}
n \\
r
\end{array}\right)\left(\begin{array}{l}
m \\
r
\end{array}\right)=\sum_{r=0}^{n}\left(\begin{array}{c}
n+m-r \\
m
\end{array}\right)\left(\begin{array}{l}
n \\
r
\end{array}\right) .
$$


Theorem 2. If $n \in \mathbb{N}_{0}$ and $m \in \mathbb{N}_{1}$, then

$$
\sum_{k=0}^{n} \frac{\left(\begin{array}{c}
m+n \\
k
\end{array}\right)}{2^{n}\left(\begin{array}{c}
m+n \\
m
\end{array}\right)}=\sum_{k=0}^{n} \frac{(-1)^{k} m\left(\begin{array}{l}
n \\
k
\end{array}\right)}{2^{k}(m+k)} .
$$

Remark. The sums in Theorem 1 are called Delannoy numbers in the literature.

\section{Combinatorial Proofs of the Main Identities}

\subsection{Proof of Theorem 1}

We show that both sides of equation (1) count the number of ways of forming two teams with $m$ and $n$ positions, respectively, formed by people of only three nationalities (American, Canadian, and Mexican) such that the total number of Canadians is $m$, and team one does not have any Americans.

Answer 1: For $0 \leq r \leq m$, there are $\left(\begin{array}{c}m \\ r\end{array}\right)$ ways to put $r$ Mexicans on team one. The remaining $m-r$ positions on team one will then be filled by Canadians. There are $\left(\begin{array}{l}n \\ r\end{array}\right)$ ways to put $r$ Canadians on team two. We then decide position by position whether each of the remaining $n-r$ positions will be filled by an American or a Mexican. There are $2^{n-r}$ ways to do this. Therefore, the are $\left(\begin{array}{c}m \\ r\end{array}\right)\left(\begin{array}{c}n \\ r\end{array}\right) 2^{n-r}$ ways to a form two teams with $r$ Mexicans on team one. Thus $\sum_{r=0}^{m}\left(\begin{array}{c}m \\ r\end{array}\right)\left(\begin{array}{l}n \\ r\end{array}\right) 2^{n-r}$ ways to form the two teams.

Answer 2: For $0 \leq r \leq n$, there are $\left(\begin{array}{l}n \\ r\end{array}\right)$ ways to put $r$ Americans on team two. Of the remain $n+m-r$ positions, choose $m$ positions to be filled by Canadians. There are $\left(\begin{array}{c}n+m-r \\ m\end{array}\right)$ ways to do this. We then assign Mexicans to the remain $n-r$ positions. Thus
$\sum_{r=0}^{n}\left(\begin{array}{c}n+m-r \\ m\end{array}\right)\left(\begin{array}{l}n \\ r\end{array}\right)$ ways to form the two teams. Hence the identity follows.

\subsection{Proof of Theorem 2}

We first divide both sides of equation (2) by $2^{n}$ and split up the sum on the right to get an equivalent identity:

$$
\begin{aligned}
\frac{\sum_{k=0}^{n}\left(\begin{array}{c}
m+n \\
k
\end{array}\right)}{4^{n}\left(\begin{array}{c}
m+n \\
m
\end{array}\right)} & =\frac{\sum_{k=0}^{n} \frac{(-1)^{k}\left(\begin{array}{l}
n \\
k
\end{array}\right) m}{2^{k}(m+k)}}{2^{n}}=\frac{2 \sum_{k=0}^{\left\lfloor\frac{n}{2}\right\rfloor} \frac{\left(\begin{array}{c}
n \\
2^{2 k}
\end{array}\right) m}{(m+2 k)}-\sum_{k=0}^{n} \frac{\left(\begin{array}{l}
n \\
k
\end{array}\right) m}{2^{k}(m+k)}}{2^{n}} \\
& =\frac{\sum_{k=0}^{\left\lfloor\frac{n}{2}\right\rfloor} \frac{\left(\begin{array}{c}
n \\
2 k
\end{array}\right) m}{2^{2 k}(m+2 k)}}{2^{n-1}}-\frac{\sum_{k=0}^{n} \frac{\left(\begin{array}{c}
n \\
2^{k}
\end{array}\right) m}{2^{n}}}{2^{n}}
\end{aligned}
$$

We break the proof of this identity into the following interesting lemmas. 
Lemma 1. If $n \in \mathbb{N}_{0}$ and $m \in \mathbb{N}_{1}$, then

$$
\frac{\sum_{k=0}^{n} \frac{\left(\begin{array}{c}
n \\
k
\end{array}\right) m}{2^{k}(m+k)}}{2^{n}}=\left(\frac{3}{4}\right)^{n}\left(1-\sum_{k=1}^{n} \frac{2^{k-1}\left(\begin{array}{c}
m+n-k \\
m
\end{array}\right)}{3^{k}\left(\begin{array}{c}
m+n \\
m
\end{array}\right)}\right)
$$

Proof. Suppose we have $m+n$ tiles divided into two groups, the first group containing the first $m$ and the second containing the last $n$. We color each of the first $m$ tiles green. Then we randomly color each of the $n$ remaining tiles either yellow or blue, with each color equally likely. Then for the $n$ tiles, colored yellow or blue, we randomly choose not to change the color, or repaint it red, with each action being equally likely. There are $3^{n}$ possible final colorings of the $m+n$ tiles, but $4^{n}$ ways to pick the colors of all $m+n$ tiles and the previous colorings of the red tiles, each of which are equally likely events.

We claim that if we randomly color the $m+n$ tiles this way, and randomly select one of the non-red tiles from all $m+n$ tiles, then the probability that there are no blue tiles showing and that the tile we selected is green is equal to both

$$
\frac{\sum_{k=0}^{n} \frac{\left(\begin{array}{c}
n \\
k
\end{array}\right) m}{2^{k}(m+k)}}{2^{n}} \text { and }\left(\frac{3}{4}\right)^{n}\left(1-\sum_{k=1}^{n} \frac{2^{k-1}\left(\begin{array}{c}
m+n-k \\
m
\end{array}\right)}{3^{k}\left(\begin{array}{c}
m+n \\
m
\end{array}\right)}\right) \text {. }
$$

There are $2^{n}$ ways to pick the set of red tiles. Each of the $n$ tiles can be red or not red, giving a $\frac{1}{2}$ probability of becoming red. Suppose that there are $k$ yellow and blue tiles (thus $n-k$ red tiles). Then the probability that there are no blue tiles and we pick a green tile is $\frac{m}{2^{k}(m+k)}$. There are $\left(\begin{array}{l}n \\ k\end{array}\right)$ ways of picking $n-k$ red tiles. Therefore, summing over $k$, the probability that there are no blue tiles and we pick a green tile is

$$
\frac{\sum_{k=0}^{n} \frac{\left(\begin{array}{l}
n \\
k
\end{array}\right) m}{2^{k}(m+k)}}{2^{n}}
$$

Now we calculate the following probabilities: the probability that there are no blue tiles and the probability that we pick a green tile given that there are no blue tiles.

For each non-green tile, there is a $\frac{1}{2}$ chance the tile ends up red, and a $\frac{1}{2}$ chance that it is blue if it is not red. So each tile has a $\frac{1}{4}$ chance of becoming blue, and a $\frac{3}{4}$ chance of not being blue. So clearly the probability that there are no blue tiles is $\left(\frac{3}{4}\right)^{n}$.

Note that, as shown above, for any individual tile the probability that it is yellow is $\frac{1}{4}$, the probability that it is blue is $\frac{1}{4}$, and the probability that it is red is $\frac{1}{2}$. So if we know only that a tile is not blue, then the probability that the tile is red is $\frac{2}{3}$.

Let $p_{2}(n)$ denote the probability that the tile we pick is green. Clearly $p_{2}(0)=1$.

Now consider the following algorithm of randomly selecting a non-red tile. We randomly select one of the $m+n$ tiles. If it is not red it is our tile and the algorithm terminates. If 
the tile is red we throw it out and repeat the algorithm on the remaining $m+n-1$ tiles. This algorithm has at most $n+1$ steps so it always terminates by choosing a non-red tile. At every step of the algorithm, each non-red tile is equally likely to be chosen, so this algorithm randomly selects a non-red tile with each non-red tile equally likely. We use this algorithm to recursively calculate $p_{2}(n)$.

On the first step there is a $\frac{m}{m+n}$ chance we pick a green tile. There is a $\frac{n}{m+n}$ chance that we pick a non-green tile, and a $\frac{2}{3}$ chance that tile is red. Repeating the algorithm with $n+1$ non-green tiles, we get

$$
p_{2}(n)=\frac{m}{m+n}+\frac{n}{m+n} \frac{2}{3} p_{2}(n-1) .
$$

Let $q_{2}(n)=1-p_{2}(n)$. Then $q_{2}(0)=0$, and

$$
p_{2}(n)=1-\frac{n}{m+n}+\frac{n}{m+n} \frac{2}{3} p_{2}(n-1) .
$$

Thus

$$
q_{2}(n)=\frac{n}{m+n}\left(1-\frac{2}{3} p_{2}(n-1)\right)=\frac{n}{m+n}\left(\frac{1}{3}+\frac{2}{3} q_{2}(n-1)\right) .
$$

It is clear by induction that

$$
q_{2}(n)=\sum_{k=1}^{n} \frac{2^{k-1}\left(\begin{array}{c}
m+n-k \\
m
\end{array}\right)}{3^{k}\left(\begin{array}{c}
m+n \\
m
\end{array}\right)}
$$

Therefore, the probability that we pick a green tile given that there are no blue tiles is

$$
1-\sum_{k=1}^{n} \frac{2^{k-1}\left(\begin{array}{c}
m+n-k \\
m
\end{array}\right)}{3^{k}\left(\begin{array}{c}
m+n \\
m
\end{array}\right)}
$$

and the probability that there are no blue tiles and that we pick a green tile is

$$
\left(\frac{3}{4}\right)^{n}\left(1-\sum_{k=1}^{n} \frac{2^{k-1}\left(\begin{array}{c}
m+n-k \\
m
\end{array}\right)}{3^{k}\left(\begin{array}{c}
m+n \\
m
\end{array}\right)}\right)
$$

Hence,

$$
\frac{\sum_{k=0}^{n} \frac{\left(\begin{array}{c}
n \\
k
\end{array}\right) m}{2^{k}(m+k)}}{2^{n}}=\left(\frac{3}{4}\right)^{n}\left(1-\sum_{k=1}^{n} \frac{2^{k-1}\left(\begin{array}{c}
m+n-k \\
m
\end{array}\right)}{3^{k}\left(\begin{array}{c}
m+n \\
m
\end{array}\right)}\right) .
$$

We now seek to evaluate $\frac{\sum_{k=0}^{\left\lfloor\frac{n}{2}\right\rfloor} \frac{\left(\begin{array}{c}n \\ 2 k\end{array}\right) m}{2^{2 k}(m+2 k)}}{2^{n-1}}$.

Assume $n \geq 1$, (when $n=0$ we can directly calculate the desired identity). Suppose we have $m+n$ tiles divided into two groups, the first group containing the first $m$ and the 
second containing the last $n$. We color each of the first $m$ tiles green. Then we randomly color each of the $n$ remaining tiles either yellow or blue, with each equally likely. Select randomly a subset with an even number of elements from the $n$ blue/yellow tiles under the following conditions: all such subsets are equally likely, leave the color of every tile in the selected subset unchanged and change the color of the remaining tiles in the blue/yellow tiles to red. We claim that if we randomly color the tiles this way, and then randomly select one of the non-red tiles, the probability that there are no blue tiles showing and that the tile we selected is green is equal to

$$
\frac{\sum_{k=0}^{\left\lfloor\frac{n}{2}\right\rfloor} \frac{\left(\begin{array}{c}
n \\
2 k
\end{array}\right) m}{2^{2 k}(m+2 k)}}{2^{n-1}} \text { and }\left(\frac{1}{4^{n}}\right)\left(3^{n}+1-\sum_{k=1}^{n-1} 2^{k-1} \frac{\left(\begin{array}{c}
m+n-k \\
m
\end{array}\right)}{\left(\begin{array}{c}
m+n \\
m
\end{array}\right)}\left(3^{n-k}-1\right)\right) \text {. }
$$

First, given $n$ tiles, there are $2^{n-1}$ ways to pick an even number of tiles to keep blue or yellow (this is why we require $n \geq 1$ ). If $2 k$ tiles remain blue or yellow, the probability that there are no blue tiles and that we pick a green tile is $\frac{m}{\left(m+2 k 2^{2 k}\right.}$. When we sum this probability over all $2 k$, we get that the probability that there are no blue tiles and we pick a green tile is

$$
\frac{\sum_{k=0}^{\left\lfloor\frac{n}{2}\right\rfloor} \frac{\left(\begin{array}{c}
n \\
2 k
\end{array}\right) m}{2^{2 k}(m+2 k)}}{2^{n-1}} .
$$

Now we prove the following lemma:

Lemma 2. After the above procedure, if there are no blue tiles, and we pick a random non-green tile, the probability that we get a red tile is $2\left(\frac{1+3^{n-1}}{1+3^{n}}\right)$.

Proof: Let $r(n)$ denote the desired probability. $n r(n)$ equals the expected value of the number of red tiles. Thus,

$$
n r(n)=\frac{\sum_{k=0}^{\left\lfloor\frac{n}{2}\right\rfloor}(n-2 k)\left(\begin{array}{c}
n \\
2 k
\end{array}\right) 2^{n-2 k}}{\sum_{k=0}^{\left\lfloor\frac{n}{2}\right\rfloor} 2^{n-2 k}\left(\begin{array}{c}
n \\
2 k
\end{array}\right)} .
$$

Hence,

$$
r(n)=\frac{\sum_{k=0}^{\left\lfloor\frac{n}{2}\right\rfloor}\left(\begin{array}{c}
n-1 \\
2 k
\end{array}\right) 2^{n-2 k}}{\sum_{k=0}^{\left\lfloor\frac{n}{2}\right\rfloor}\left(\begin{array}{c}
n \\
2 k
\end{array}\right) 2^{n-2 k}}=2 \frac{\sum_{k=0}^{\left\lfloor\frac{n-1}{2}\right\rfloor}\left(\begin{array}{c}
n-1 \\
2 k
\end{array}\right) 2^{n-1-2 k}}{\sum_{k=0}^{\left\lfloor\frac{n}{2}\right\rfloor}\left(\begin{array}{c}
n \\
2 k
\end{array}\right) 2^{n-2 k}}=\frac{2 a_{n-1}}{a_{n}},
$$

where $a_{n}=\sum_{k=0}^{\left\lfloor\frac{n}{2}\right\rfloor}\left(\begin{array}{c}n \\ 2 k\end{array}\right) 2^{n-2 k}$.

Now using the identity $\left(\begin{array}{l}A \\ B\end{array}\right) 2^{A-B}=\sum_{k=B}^{A}\left(\begin{array}{l}A \\ k\end{array}\right)\left(\begin{array}{l}k \\ B\end{array}\right)$ we get

$$
a_{n}=\sum_{k=0}^{\left\lfloor\frac{n}{2}\right\rfloor}\left(\begin{array}{c}
n \\
2 k
\end{array}\right) 2^{n-2 k}=\sum_{k=0}^{\left\lfloor\frac{n}{2}\right\rfloor} \sum_{j=2 k}^{n}\left(\begin{array}{c}
n \\
j
\end{array}\right)\left(\begin{array}{c}
j \\
2 k
\end{array}\right) .
$$


By reversing the order of summation,

$$
a_{n}=\sum_{j=0}^{n} \sum_{k=0}^{\left\lfloor\frac{j}{2}\right\rfloor}\left(\begin{array}{l}
n \\
j
\end{array}\right)\left(\begin{array}{c}
j \\
2 k
\end{array}\right)=\sum_{j=0}^{n}\left(\begin{array}{l}
n \\
j
\end{array}\right) \sum_{k=0}^{\left\lfloor\frac{j}{2}\right\rfloor}\left(\begin{array}{c}
j \\
2 k
\end{array}\right) .
$$

Now for $j \geq 1, \sum_{k=0}^{\left\lfloor\frac{j}{2}\right\rfloor}\left(\begin{array}{c}j \\ 2 k\end{array}\right)=2^{j-1}$. Therefore,

$$
a_{n}=\sum_{j=1}^{n}\left(\begin{array}{c}
n \\
j
\end{array}\right) 2^{j-1}+1=\sum_{j=0}^{n}\left(\begin{array}{c}
n \\
j
\end{array}\right) 2^{j-1}+\frac{1}{2}=\frac{\sum_{j=0}^{n}\left(\begin{array}{c}
n \\
j
\end{array}\right) 2^{j}+1}{2}
$$

By the binomial theorem, it follows that $a_{n}=\frac{3^{n}+1}{2}$. Thus, $r(n)=2\left(\frac{1+3^{n-1}}{1+3^{n}}\right)$.

Lemma 3. Under the restriction that the number of blue or yellow tiles is even, the probability that there are no blue tiles and that we pick a green tile is

$$
\left(\frac{1}{4^{n}}\right)\left(3^{n}+1-\sum_{k=1}^{n-1} 2^{k-1} \frac{\left(\begin{array}{c}
m+n-k \\
m
\end{array}\right)}{\left(\begin{array}{c}
m+n \\
m
\end{array}\right)}\left(3^{n-k}-1\right)\right)
$$

Proof. This probability is equal to the product of probability that there are no blue tiles and the probability that we pick a green tile, given that there are no blue tiles.

The probability that there are no blue tiles is

$$
\frac{\sum_{k=0}^{\left\lfloor\frac{n}{2}\right\rfloor}\left(\begin{array}{c}
n \\
2 k
\end{array}\right) \frac{1}{2^{2 k}}}{2^{n-1}}=\frac{\sum_{k=0}^{\left\lfloor\frac{n}{2}\right\rfloor}\left(\begin{array}{c}
n \\
2 k
\end{array}\right) 2^{n-2 k}}{2^{2 n-1}}=\frac{a_{n}}{2^{2 n-1}}=\frac{3^{n}+1}{4^{n}} .
$$

Now let $p_{1}(n)$ be the probability that we pick a green tile given that there are no blue tiles. The total probability that we pick a green tile and there are no blue tiles is $\left(\frac{3^{n}+1}{4^{n}}\right) p_{1}(n)$. Clearly $p_{1}(0)=1$, but also note that $p_{1}(1)=1$ since when $n=1$ we must have 1 red tile and 0 blue or yellow tiles.

We use the same algorithm as in the first lemma to randomly pick a green or yellow tile. We pick a random tile: if it is not red we are done; if it is red, we toss it out and repeat the algorithm. On the first step of the algorithm one of the following happens: we pick a green tile with $\frac{m}{m+n}$ probability, we pick a red tile with $r(n) \frac{n}{m+n}$ chance and repeat the algorithm; otherwise we have picked a yellow tile and the algorithm terminates. Thus, we have

$$
p_{1}(n)=\frac{m}{m+n}+r(n) \frac{n}{m+n} p_{1}(n-1) .
$$


Let $q_{1}(n)=1-p_{1}(n)$. By Lemma 2, we have

$$
\begin{aligned}
p_{1}(n) & =\frac{m}{m+n}+2\left(\frac{1+3^{n-1}}{1+3^{n}}\right) \frac{n}{m+n} p_{1}(n-1) \\
0 & =1-\frac{n}{n+m}-p_{1}(n)+\left(\frac{2+3^{n-1} 2}{1+3^{n}}\right) \frac{n}{m+n} p_{1}(n-1) .
\end{aligned}
$$

Therefore,

$$
\begin{aligned}
q_{1}(n) & =\frac{n}{n+m}\left(\frac{3^{n-1}-1}{3^{n}+1}+\left(\frac{2+3^{n-1} 2}{1+3^{n}}\right) q_{1}(n-1)\right) \\
& =\left(\frac{n}{n+m}\right)\left(\frac{3^{n-1}-1}{3^{n}+1}\right)+\left(\frac{n}{n+m}\right)\left(\frac{2+3^{n-1} 2}{1+3^{n}} q_{1}(n-1)\right) .
\end{aligned}
$$

From $p_{1}(1)=1$ we know $q_{1}(1)=0$, and an easy induction implies

$$
q_{1}(n)=\sum_{k=1}^{n-1} 2^{k-1} \frac{\left(\begin{array}{c}
m+n-k \\
m
\end{array}\right)}{\left(\begin{array}{c}
m+n \\
m
\end{array}\right)} \frac{3^{n-k}-1}{3^{n}+1} .
$$

Hence, the probability that there are no blue tiles and that we pick a green tile is

$$
\begin{aligned}
\left(\frac{3^{n}+1}{4^{n}}\right) p_{1}(n) & =\left(\frac{3^{n}+1}{4^{n}}\right)\left(1-\sum_{k=1}^{n-1} 2^{k-1} \frac{\left(\begin{array}{c}
m+n-k \\
m
\end{array}\right)}{\left(\begin{array}{c}
m+n \\
m
\end{array}\right)} \frac{3^{n-k}-1}{3^{n}+1}\right) \\
& =\left(\frac{1}{4^{n}}\right)\left(3^{n}+1-\sum_{k=1}^{n-1} 2^{k-1} \frac{\left(\begin{array}{c}
m+n-k \\
m
\end{array}\right)}{\left(\begin{array}{c}
m+n \\
m
\end{array}\right)}\left(3^{n-k}-1\right)\right) .
\end{aligned}
$$

This proves that

$$
\frac{\sum_{k=0}^{\left\lfloor\frac{n}{2}\right\rfloor} \frac{\left(\begin{array}{c}
n \\
2 k
\end{array}\right) m}{2^{2 k}(m+2 k)}}{2^{n-1}}=\left(\frac{1}{4^{n}}\right)\left(3^{n}+1-\sum_{k=1}^{n-1} 2^{k-1} \frac{\left(\begin{array}{c}
m+n-k \\
m
\end{array}\right)}{\left(\begin{array}{c}
m+n \\
m
\end{array}\right)}\left(3^{n-k}-1\right)\right) .
$$


Combining all lemmas, we get

$$
\begin{aligned}
& \frac{\sum_{k=0}^{n} \frac{(-1)^{k}\left(\begin{array}{l}
n \\
k
\end{array}\right) m}{2^{k}(m+k)}}{2^{n}}=\frac{\sum_{k=0}^{\left\lfloor\frac{n}{2}\right\rfloor} \frac{\left(\begin{array}{c}
n \\
2 k
\end{array}\right) m}{2^{2 k}(m+2 k)}}{2^{n-1}}-\frac{\sum_{k=0}^{n} \frac{\left(\begin{array}{c}
n \\
k
\end{array}\right) m}{2^{k}(m+k)}}{2^{n}} \\
& =\left(\frac{1}{4^{n}}\right)\left(3^{n}+1-\sum_{k=1}^{n-1} 2^{k-1} \frac{\left(\begin{array}{c}
m+n-k \\
k
\end{array}\right)}{\left(\begin{array}{c}
m+n \\
m
\end{array}\right)}\left(3^{n-k}-1\right)\right) \\
& -\left(\frac{3}{4}\right)^{n}\left(1-\sum_{k=1}^{n} \frac{2^{k-1}\left(\begin{array}{c}
m+n-k \\
m
\end{array}\right)}{3^{k}\left(\begin{array}{c}
m+n \\
m
\end{array}\right)}\right) \\
& =\frac{1-\sum_{k=1}^{n-1} 2^{k-1} \frac{\left(\begin{array}{c}
m+n-k \\
m
\end{array}\right)}{\left(\begin{array}{c}
m+n \\
m
\end{array}\right)}\left(3^{n-k}-1\right)+\sum_{k=1}^{n} 2^{k-1} \frac{\left(\begin{array}{c}
m+n-k \\
m
\end{array}\right)}{\left(\begin{array}{c}
m+n \\
m
\end{array}\right)} 3^{n-k}}{4^{n}} \\
& =\frac{\left(\begin{array}{c}
m+n \\
m
\end{array}\right)-\sum_{k=1}^{n-1} 2^{k-1}\left(\begin{array}{c}
m+n-k \\
m
\end{array}\right)\left(3^{n-k}-1\right)+\sum_{k=1}^{n} 2^{k-1}\left(\begin{array}{c}
m+n-k \\
m
\end{array}\right) 3^{n-k}}{4^{n}\left(\begin{array}{c}
m+n \\
m
\end{array}\right)} \\
& =\frac{\left(\begin{array}{c}
m+n \\
m
\end{array}\right)+\sum_{k=1}^{n-1} 2^{k-1}\left(\begin{array}{c}
m+n-k \\
m
\end{array}\right)+2^{n-1}}{4^{n}\left(\begin{array}{c}
m+n \\
m
\end{array}\right)} \\
& =\frac{\left(\begin{array}{c}
m+n \\
m
\end{array}\right)+\sum_{k=1}^{n} 2^{k-1}\left(\begin{array}{c}
m+n-k \\
m
\end{array}\right)}{4^{n}\left(\begin{array}{c}
m+n \\
m
\end{array}\right)} \\
& =\frac{\left(\begin{array}{c}
m+n \\
m
\end{array}\right)+\sum_{k=0}^{n-1} 2^{k}\left(\begin{array}{c}
m+n-1-k \\
m
\end{array}\right)}{4^{n}\left(\begin{array}{c}
m+n \\
m
\end{array}\right)} \text {. }
\end{aligned}
$$

Lemma 4. $\sum_{k=0}^{n-1} 2^{k}\left(\begin{array}{c}m+n-1-k \\ m\end{array}\right)=\sum_{k=0}^{n-1}\left(\begin{array}{c}m+n \\ k\end{array}\right)$.

Proof: We claim both quantities count the number of ways to pick at least $m+1$ integers from the first $m+n$ integers. If we sum over the number of objects picked, we get

$$
\sum_{k=m+1}^{m+n}\left(\begin{array}{c}
m+n \\
k
\end{array}\right)
$$

Reversing the order of summation yields

$$
\sum_{k=0}^{n-1}\left(\begin{array}{c}
m+n \\
m+n-k
\end{array}\right)=\sum_{k=0}^{n-1}\left(\begin{array}{c}
m+n \\
k
\end{array}\right)
$$

If we sum over the placement of the $(m+1)$-th relatively smallest integer in the set we get

$$
\sum_{k=m+1}^{m+n} 2^{m+n-k}\left(\begin{array}{c}
k-1 \\
m
\end{array}\right)
$$


Reversing the order of summation yields

$$
\sum_{k=0}^{n-1} 2^{k}\left(\begin{array}{c}
m+n-k-1 \\
m
\end{array}\right)
$$

Therefore,

$$
\sum_{k=0}^{n-1} 2^{k}\left(\begin{array}{c}
m+n-1-k \\
m
\end{array}\right)=\sum_{k=0}^{n-1}\left(\begin{array}{c}
m+n \\
k
\end{array}\right)
$$

Now we have

$$
\frac{\sum_{k=0}^{n} \frac{(-1)^{k}\left(\begin{array}{l}
n \\
k
\end{array}\right) m}{2^{k}(m+k)}}{2^{n}}=\frac{\left(\begin{array}{c}
m+n \\
m
\end{array}\right)+\sum_{k=0}^{n-1}\left(\begin{array}{c}
m+n \\
k
\end{array}\right)}{4^{n}\left(\begin{array}{c}
m+n \\
m
\end{array}\right)}=\frac{\sum_{k=0}^{n}\left(\begin{array}{c}
m+n \\
k
\end{array}\right)}{4^{n}\left(\begin{array}{c}
m+n \\
m
\end{array}\right)}
$$

Therefore,

$$
\sum_{k=0}^{n} \frac{(-1)^{k}\left(\begin{array}{l}
n \\
k
\end{array}\right) m}{2^{k}(m+k)}=\frac{\sum_{k=0}^{n}\left(\begin{array}{c}
m+n \\
k
\end{array}\right)}{2^{n}\left(\begin{array}{c}
m+n \\
m
\end{array}\right)}
$$

This completes the proof of Theorem 2 .

\section{Automated Proofs}

\subsection{Proof of Theorem 1}

Since the summands on the left and right sides of equation (1) vanish outside the intervals $[0, m]$ and $[0, n]$, the identity is equivalent to

$$
\sum_{r \in \mathbb{Z}} 2^{n-r}\left(\begin{array}{l}
n \\
r
\end{array}\right)\left(\begin{array}{l}
m \\
r
\end{array}\right)=\sum_{r \in \mathbb{Z}}\left(\begin{array}{c}
n+m-r \\
m
\end{array}\right)\left(\begin{array}{l}
n \\
r
\end{array}\right) .
$$

Suppressing the free parameter $m$, let us denote the left and right sides of equation (3) by $S(n)$ and $T(n)$, respectively. Let $F_{1}(n, r)$ denote the summand of $S(n)$, i.e., $F_{1}(n, r)=2^{n-r}\left(\begin{array}{c}n \\ r\end{array}\right)\left(\begin{array}{c}m \\ r\end{array}\right)$. Applying Zeilberger's algorithm to $F_{1}$, we get the recurrence equation:

$-(n+2) F_{1}(n+2, r)+(3 n+m+5) F_{1}(n+1, r)-2(n+1) F_{1}(n, r)=G_{1}(n, r+1)-G_{1}(n, r)$

where

$$
G_{1}(n, r)=2^{n+2-r} r\left(\begin{array}{c}
n+1 \\
r-1
\end{array}\right)\left(\begin{array}{c}
m \\
r
\end{array}\right) .
$$

Summing both sides of equation (4) over all integers $r$ yields

$$
-(n+2) S(n+2)+(3 n+m+5) S(n+1)-2(n+1) S(n)=0 .
$$


Now let $F_{2}(n, r)$ denote the summand of $T(n)$. Applying Zeilberger's algorithm to $F_{2}$, we get the recurrence equation:

$$
-(n+2) F_{2}(n+2, r)+(3 n+m+5) F_{2}(n+1, r)-2(n+1) F_{2}(n, r)=G_{2}(n, r+1)-G_{2}(n, r)
$$

where

$$
G_{2}(n, r)=\frac{\left(2(n+2) m+(n+2-m) r-r^{2}\right) r}{m(n+2)}\left(\begin{array}{c}
n+m-r+1 \\
m-1
\end{array}\right)\left(\begin{array}{c}
n+2 \\
r
\end{array}\right) .
$$

Summing both sides of this recurrence equation over all integers $r$ yields

$$
-(n+2) T(n+2)+(3 n+m+5) T(n+1)-2(n+1) T(n)=0 .
$$

Therefore, $S(n)$ and $T(n)$ satisfy the same linear recurrence relation. In addition, $S(0)=$ $T(0)=1$ and $S(1)=T(1)=m+2$. Thus $S(n)=T(n)$ for all $n \in \mathbb{N}_{0}$.

\subsection{Proof of Theorem 2}

Let $F(n, k)=\left(\begin{array}{c}m+n \\ k\end{array}\right)\left(\begin{array}{c}m+n \\ m-1\end{array}\right)^{-1} \frac{1}{2^{n}(n+1)}$ and $f(n)=\sum_{k=0}^{n} F(n, k)$.

Applying Zeilberger's algorithm to $F$, we get the recurrence equation:

$$
\begin{aligned}
(m+n+1) F(n+1, k)- & (n+1) F(n, k) \\
= & \frac{(k+1)\left(\begin{array}{c}
m+n \\
k+1
\end{array}\right)}{(2 k-2 m-2 n)\left(\begin{array}{c}
m+n \\
m-1
\end{array}\right) 2^{n}}-\frac{k\left(\begin{array}{c}
m+n \\
k
\end{array}\right)}{(2 k-2 m-2 n-2)\left(\begin{array}{c}
m+n \\
m-1
\end{array}\right) 2^{n}} .
\end{aligned}
$$

We sum both sides from $k=0$ to $n$ to get

$$
(m+n+1)(f(n+1)-F(n+1, n+1))-(n+1) f(n)=\frac{(n+2)\left(\begin{array}{c}
m+n \\
n+2
\end{array}\right)}{(2-2 m)\left(\begin{array}{c}
m+n \\
m-1
\end{array}\right) 2^{n}} .
$$

Using the rsolve command in Maple, we find that

$$
f(n)=\frac{2^{m}(m-1) ! n !}{(m+n) !}-\frac{1}{2^{n+1}(n+1)} \sum_{k=0}^{\infty} \frac{\left(\begin{array}{c}
m+n+k \\
k
\end{array}\right)}{\left(\begin{array}{c}
n+k+1 \\
k
\end{array}\right) 2^{k}} .
$$

Now let $T(n, k)=(-1)^{k}\left(\begin{array}{l}n \\ k\end{array}\right) \frac{1}{2^{k}(m+k)}$ and $t(n)=\sum_{k=0}^{n} T(n, k)$.

We input $T(n, k)$ into Zeilberger's algorithm to get the following recurrence:

$$
\begin{aligned}
& (-2 n-2 m-4) T(n+2, k)+(3 n+5+m) T(n+1, k)-(n+1) T(n, k) \\
& =\frac{2(k+1)(-1)^{k+1}\left(\begin{array}{c}
n \\
k+1
\end{array}\right)(n+1)}{(k-n)(k-n-1) 2^{k+1}}-\frac{2 k(-1)^{k}\left(\begin{array}{c}
n \\
k
\end{array}\right)(n+1)}{(k-n-1)(k-n-2) 2^{k}} .
\end{aligned}
$$


We sum both sides from $k=0$ to $n-1$ to get

$$
\begin{aligned}
& (-2 n-2 m-4)(t(n+2)-T(n+2, n+2)-T(n+2, n+1)-T(n+2, n)) \\
& \quad+(3 n+5+m)(t(n+1)-T(n+1, n+1)-T(n+1, n))-(n+1)(t(n)-T(n, n)) \\
& =\frac{n(-1)^{n}(n+1)}{2^{n}} .
\end{aligned}
$$

Using the rsolve command in Maple, we find that

$$
t(n)=\frac{2^{m}(m-1) ! n !}{(m+n) !}-\frac{1}{2^{n+1}(n+1)} \sum_{k=0}^{\infty} \frac{\left(\begin{array}{c}
m+n+k \\
k
\end{array}\right)}{\left(\begin{array}{c}
n+k+1 \\
k
\end{array}\right) 2^{k}}
$$

Therefore, $f(n)=t(n)$ for all $n \in \mathbb{N}_{0}$.

Remark. Those recurrences pertinent to Section 4 are automatically generated by the Maple package EKHAD which is freely available from [3]. Alternatively, one can also use the built-in SumTools package in Maple that contains programs that implement Zeilberger's algorithm.

\section{$5 \quad$ Proofs with Generatingfunctionology}

\subsection{Proof of Theorem 1}

We show that the left and right sides of equation (1) represent the coefficient of $x^{m}$ in the expansion of $F(x)=(x+2)^{n}(x+1)^{m}$. Now

$$
\begin{aligned}
F(x) & =\left(\sum_{k=0}^{\infty}\left(\begin{array}{l}
n \\
k
\end{array}\right) 2^{n-k} x^{k}\right)\left(\sum_{k=0}^{\infty}\left(\begin{array}{l}
m \\
k
\end{array}\right) x^{k}\right) \\
& =\sum_{k=0}^{\infty}\left(\sum_{r=0}^{k}\left(\begin{array}{l}
n \\
r
\end{array}\right) 2^{n-r}\left(\begin{array}{c}
m \\
k-r
\end{array}\right)\right) x^{k}
\end{aligned}
$$

Therefore, $\left[x^{m}\right] F(x)=\sum_{r=0}^{m}\left(\begin{array}{l}n \\ r\end{array}\right) 2^{n-r}\left(\begin{array}{c}m \\ m-r\end{array}\right)=\sum_{r=0}^{m} 2^{n-r}\left(\begin{array}{l}n \\ r\end{array}\right)\left(\begin{array}{c}m \\ r\end{array}\right)$.

But $F(x)$ can also be written as

$F(x)=(1+(x+1))^{n}(x+1)^{m}=\sum_{k=0}^{n}\left(\begin{array}{l}n \\ k\end{array}\right)(x+1)^{n+m-k}=\sum_{k=0}^{\infty} \sum_{j=0}^{n+m-k}\left(\begin{array}{l}n \\ k\end{array}\right)\left(\begin{array}{c}n+m-k \\ j\end{array}\right) x^{j}$.

Therefore, $\left[x^{m}\right] F(x)=\sum_{k=0}^{n}\left(\begin{array}{l}n \\ k\end{array}\right)\left(\begin{array}{c}n+m-k \\ m\end{array}\right)$. Hence the identity follows. 


\subsection{Proof of Theorem 2}

Equation (2) is equivalent to

$$
\sum_{k=0}^{n}\left(\begin{array}{c}
m+n \\
k
\end{array}\right)=\sum_{k=0}^{n}(-1)^{k}\left(\begin{array}{c}
m+n \\
m
\end{array}\right)\left(\begin{array}{l}
n \\
k
\end{array}\right) \frac{m}{m+k} 2^{n-k}
$$

Suppressing the free parameter $m$, let $p_{n}$ and $q_{n}$ denote the left and right sides of equation (5), respectively. Let $P(x)=\sum_{n \geq 0} p_{n} x^{n}$ and $Q(x)=\sum_{n \geq 0} q_{n} x^{n}$. We need to show that $P(x)=Q(x)$. Pascal's relation $\left(\begin{array}{c}n+m \\ k\end{array}\right)=\left(\begin{array}{c}n+m-1 \\ k\end{array}\right)+\left(\begin{array}{c}n+m-1 \\ k-1\end{array}\right)$ implies that $p_{n}=$ $\left(\begin{array}{c}n+m-1 \\ n\end{array}\right)+2 p_{n-1}$. That means $P(x)-2 x P(x)=\sum_{n \geq 0}\left(\begin{array}{c}n+m-1 \\ n\end{array}\right) x^{n}=\frac{1}{(1-x)^{m}}$, hence $P(x)=\frac{1}{(1-2 x)(1-x)^{m}}$.

Swapping the order of summation in $Q(x)$ as $\sum_{k \geq 0} \sum_{n \geq k}$ we get

$$
\begin{aligned}
Q(x) & =\sum_{k \geq 0} \frac{(-1)^{k} m}{(m+k) 2^{k}} \sum_{n \geq k}\left(\begin{array}{c}
n+m \\
m
\end{array}\right)\left(\begin{array}{l}
n \\
k
\end{array}\right)(2 x)^{n} \\
& =\sum_{k \geq 0} \frac{(-1)^{k} m}{(m+k) 2^{k}}\left(\begin{array}{c}
m+k \\
k
\end{array}\right) \sum_{n \geq k}\left(\begin{array}{l}
m+n \\
m+k
\end{array}\right)(2 x)^{n} \\
& =\sum_{k \geq 0} \frac{(-1)^{k} m}{(m+k) 2^{k}}\left(\begin{array}{c}
m+k \\
k
\end{array}\right) \frac{(2 x)^{k}}{(1-2 x)^{k}} \\
& =\frac{1}{(1-2 x)^{m+1}} \sum_{k \geq 0}\left(\begin{array}{c}
m+k-1 \\
k
\end{array}\right) \frac{(-x)^{k}}{(1-2 x)^{k}} \\
& =\frac{1}{(1-2 x)^{m+1}}\left(1+\frac{x}{1-2 x}\right)^{-m} \\
& =P(x) .
\end{aligned}
$$

Remark. The expansion $\frac{1}{(1-y)^{m}}=\sum_{n \geq 0}\left(\begin{array}{c}m+n-1 \\ n\end{array}\right) y^{n}$ has been utilized repeatedly.

\section{Acknowledgements}

We would like to thank the anonymous referee for providing us with constructive comments and suggestions and for providing us a generatingfunctionology proof of Theorem 2. This work was supported by the National Security Agency (NSA) under Grant No. H98230- 10-1-0222, which funds the Summer Undergraduate Research Institute in Experimental Mathematics (SURIEM) at Michigan State University (MSU). The authors would like to thank NSA and MSU for their generous support. 


\section{References}

[1] A. T. Benjamin and J. J. Quinn, Proofs that Really Count: The Art of Combinatorial Proof, The Mathematical Association of America, 2003.

[2] M. Bóna, A Walk Through Combinatorics: An Introduction to Enumeration and Graph Theory, 2nd ed., 2006.

[3] EKHAD, a MAPLE package by Doron Zeilberger, http://www.math.rutgers.edu/ $\sim$ zeilberg/.

[4] W. Koepf, Hypergeometric Summation: An Algorithmic Approach to Summation and Special Function Identities, AMS, 1998.

[5] M. Petkovšek, H. S. Wilf and D. Zeilberger, $A=B$, A. K. Peters, Wellesley, Massachusetts, 1996.

[6] A. Tefera, What is a Wilf-Zeilberger Pair?, Notices of the American Mathematical Society, Vol. 57, No. 4, 2010, pp.508-509.

[7] H. S. Wilf, generatingfunctionology, Academic Press, 2nd ed., 1993. 